that the area of potential addressment with a suburethral sling is not surgically altered through their urethroplasty approach.

\title{
Reference
}

1. Petrou SP, Brown JA, Blaivas JG: Suprameatal transvaginal urethrolysis. J Urol. 1999; 161: 1268-71.

Dr. Steven P. Petrou

Associate Professor of Urology

Chief of Surgery, St. Luke's Hospital

Associate Dean, Mayo School of Graduate Medical Education

Jacksonville, Florida, USA

\section{PEDIATRIC UROLOGY}

\section{Bladder Reservoir Function in Children with Monosymptomatic Nocturnal Enuresis and Healthy Controls}

Hagstroem S, Kamperis K, Rittig S, Djurhuus JC

Clinical Institute, University of Aarhus and Department of Pediatrics, Skejby University Hospital, Denmark

J Urol. 2006;176:759-63

Purpose: We investigated bladder reservoir function in children with monosymptomatic nocturnal enuresis and in healthy controls.

Materials and Methods: A total of 18 children with monosymptomatic nocturnal enuresis and 119 controls who were 7 to 13 years old were recruited. Children completed frequency volume charts and measurements of nocturnal urine production. Mean diuresis in the period preceding each voiding was calculated. Those with enuresis were grouped according to bladder capacity and hospitalized for 4 nights, including a baseline night and 3 with an oral water load. Enuresis volumes and post-void residual volume were estimated, allowing the calculation of bladder volume at the time of enuresis.

Results: Nine children with monosymptomatic nocturnal enuresis were characterized as having normal bladder capacity and 9 had decreased bladder capacity. We found large intra-individual variability in daytime voided volume in all 3 groups of participants. Children with enuresis and small bladder capacity generally voided with volumes close to maximal voided volume. A total of 93 enuresis episodes were recorded. Large intra-individual variability was seen in bladder volume at enuresis and it was lower than maximal voided volume in more than $50 \%$ of episodes. Variability in bladder volume at enuresis was greatest in the patient group with decreased bladder capacity. We found a significant correlation between diuresis and bladder capacity in all groups during the day and night.

Conclusions: There is a great intra-individual diurnal variability in voided volume in children with enuresis and in healthy children. Enuresis seems to occur at bladder volumes that are smaller and larger than the maximal voided volume obtained from voiding charts.

\section{Editorial Comment}

The authors attempted to measure bladder size in children using voiding diaries and, in enuretic children, observed voiding overnight in hospital, including after water loading. They found that about half of children 
with enuresis had smaller than expected functional bladder capacity. However, there was a large intra-individual variability, both in normal controls and children with enuresis. Similarly, looking at nocturnal enuretic episodes in particular, there was wide variation and at least $50 \%$ of episodes occurred at volumes less than the maximal voided volume during the day. Finally, water loading appeared to increase functional bladder size.

These findings are of considerable interest. First, the finding of smaller then expected bladder capacity suggests that some of the children may well benefit from anticholinergic therapy. Of course, this has been recognized in the past and the children who do respond are limited. What is interesting, yet disconcerting is the wide variation. Though not particularly surprising, as many social and behavioral factors can influence voiding function, this calls into question the usefulness of short-term voiding diaries. These diaries are the initial noninvasive assessment in most children with voiding problems, yet are clearly highly flawed as a tool to estimate bladder capacity. Also concerning is the finding that for control children the largest voided volume was usually the first void in the AM. In children with nocturnal enuresis, in whom the diary would be most useful, this measurement will clearly not be comparable (as the child will have voided during the night). This again limits the usefulness of the voiding diary. An interesting finding that might have therapeutic benefit is the determination that water loading could, over a few days, lead to larger voided volumes. If so, could morning water loading be of benefit to nocturnal enuresis? Further studies are needed.

\author{
Dr. Barry A. Kogan \\ Chief and Professor of Urology and Pediatrics \\ Albany Medical College \\ Albany, New York, USA
}

\title{
The Impact of Attention Deficit Hyperactivity Disorders on Brainstem Dysfunction in Nocturnal Enuresis
} Baeyens D, Roeyers H, Hoebeke P, Antrop I, Mauel R, Walle JV

Department of Psychology, Developmental Disorders, Ghent University, Belgium

J Urol. 2006; 176: 744-8

Purpose: In a specialized university setting the prevalence of attention deficit hyperactivity disorder in general and particularly the inattentive subtype attention deficit hyperactivity disorder of the predominantly inattentive subtype is highly increased. We replicated previous research findings that enuresis is associated with a brainstem deficit and investigated the impact of attention deficit hyperactivity disorder on this brainstem deficit in enuresis.

Materials and Methods: Electromyography recorded startle eye blink modification with and without attentional modulation was used to measure brainstem functioning in 158 children between 6 and 12 years old. Performance in 3 enuresis groups, including children with enuresis, enuresis plus attention deficit hyperactivity disorder of the predominantly inattentive subtype and enuresis plus attention deficit hyperactivity disorder combined, respectively, was compared with that in normally developing controls and in children with attention deficit hyperactivity disorder subtypes without enuresis.

Results: In an automatic attentional task the enuresis groups showed decreased brainstem inhibition compared to that in the control and attention deficit hyperactivity disorder groups ( $\mathrm{p}<0.006)$. In a controlled attentional task children with and without enuresis who had attention deficit hyperactivity disorder of the predominantly inattentive subtype were unable to show attentional modulation in all age groups ( $\mathrm{p}<0.02)$.

Conclusions: Startle eye blink modification research reveals a brainstem inhibition deficit in children with enuresis, which could explain why they are unable to remain dry at night. When additional attention is allocated to specific trials in the task, children with attention deficit hyperactivity disorder of the predominantly inatten- 
tive subtype fail to optimize sensory gating. With respect to enuresis, this could result in an identification problem of bladder signals, leading to an inadequate or absent arousal effect in attention deficit hyperactivity disorder of the predominantly inattentive subtype.

\section{Editorial Comment}

The authors use Startle Eye Blink studies to compare children with enuresis to controls and to compare those with attention deficit disorders to normals. They demonstrated clear differences, with enuretic children (even those with ADHD) having much lower percentage pre-impulse inhibition of startle. In some ways but not all, the response was similar to that seen in patients with ADHD-inattentive subtype who did not have enuresis. Because Startle Eye Blink studies are a measure of brain-stem function, the results indicate that children with enuresis have a clearly documented brain stem dysfunction. In general, this dysfunction over-rides the effects of ADHD as an association with enuresis.

Nocturnal enuresis is caused by a combination of a large nocturnal urine output, small nocturnal bladder capacity and a failure of arousal. These factors all can be related to central nervous system dysfunction. Hence, it is not surprising that enuretic children might have brainstem dysfunction. This particular study is confounded a bit in that almost $30 \%$ of the enuretic group had daytime symptoms also. Since it is generally thought that diurnal enuresis has a different etiology than mono-symptomatic nocturnal enuresis, it would have been nice to have the different groups separated out. Also, the percentage pre-impulse inhibition of startle can be increased by medications, in particular methylphenidate. It would have been fascinating to test the results of methylphenidate treatment in these groups, even though it has not been shown to improve enuresis. Any divergence of effect would be helpful in understanding the pathophysiology of nocturnal enuresis better.

Studies of this type should be encouraged. The relationship between brain function and voiding function is intuitively clear, but obviously quite complex. Nonetheless, further application of modern neuroscience tools to patients with voiding function should be encouraged as these investigations will be helpful in understanding the conditions and in developing new therapeutic interventions.

Dr. Barry A. Kogan Chief and Professor of Urology and Pediatrics Albany Medical College Albany, New York, USA 